Psychological Medicine, 2000, 30, 1-9. Printed in the United Kingdom

(C) 2000 Cambridge University Press

\title{
EDITORIAL
}

\section{Personality and personality disorder: current issues and directions ${ }^{1}$}

The last decade has seen a renewed interest and research effort focused on the broad areas of personality and personality disorder. Issues preoccupying researchers include problems of classification (especially categorical versus dimensional models), the absence of theoretical models, and implications of the suggested continuity between 'normal' personality dimensions and personality disorder.

\section{CLASSIFICATION ISSUES AND THEORETICAL MODELS}

One keenly debated issue concerns the respective merits of categorical versus dimensional approaches to the assessment of personality disorder (PD). The DSM system clearly shapes current theorizing and clinical diagnostic practices in this area. According to DSM-IV (APA, 1994), personality disorder is 'an enduring pattern of inner experience and behavior that deviates markedly from the expectations of the individual's culture and is manifested in at least two of the following areas: cognition, affectivity, interpersonal functioning or impulse control'. Furthermore, the enduring pattern is required to be 'inflexible and pervasive', 'stable over time', have an 'onset in adolescence or early adulthood' and 'lead to clinically significant distress or impairment in social, occupational or other important areas of functioning'. DSM-IV lists 10 specific personality disorders that are grouped into three separate clusters based on descriptive similarities: Cluster A (an 'odd or eccentric' pattern); Cluster B ('dramatic, emotional or erratic'); and Cluster C ('anxious and fearful'). Thus, the categorical model views PDs as qualitatively distinct clinical syndromes with three higher order classes or clusters. Its limitations have been well documented, most notable being the basic assumption that a disorder is either present or absent, and as a consequence, the fostering of an arbitrary distinction between normal and abnormal personality (Shea, 1995). Thus, for individual personality disorders, reliability of diagnosis is modest at best, while reaching diagnostic criteria for one personality disorder is frequently associated with also meeting criteria for several other personality disorders. Even the DSM cluster model has resisted empirical support, with two-cluster and four-cluster solutions most commonly identified and with compulsive PD loading on any fourth cluster rather than with the anxiety cluster PDs (see Parker et al. 1998).

The contrasting dimensional view regards PDs as representing 'maladaptive variants of personality traits that merge imperceptibly into normality and into one another' (APA, 1994). This now has a strong evidence base. Thus, PD features do not form discrete categories according to a categorical model but are continuously distributed (Widiger, 1992; Livesley et al. 1994), while numerous studies have failed to identify evidence of discontinuity or a distinct point of demarcation between PDs and normal personality functioning or among the different PDs themselves (Zimmerman \& Coryell, 1990; Livesley et al. 1992; Nestadt et al. 1992).

Such evidence of continuity has been advanced by numerous theoretical dimensional models over the years. An early formulation was the Interpersonal Circumplex Model (Leary, 1957; Wiggins, 1982) which conceptualizes PDs by reference to only two orthogonal and bipolar dimensions labelled 'dominance versus submissiveness' and 'love versus hate'. Despite widespread agreement regarding the centrality of interpersonal dysfunction to any definition of PD, this model, in focusing

\footnotetext{
${ }^{1}$ Address for correspondence: Professor Gordon Parker, School of Psychiatry, Prince of Wales Hospital, Randwick, NSW 2031, Australia.
} 
on the interpersonal component only, cannot provide a complete framework for understanding the PDs.

More cogent frameworks have emerged from attempts to define normal personality functioning, such as the Five Factor Model or FFM (Widiger \& Costa, 1994) and Cloninger's biopsychosocial model (Cloninger, 1987), as well as from studying patients with PDs, and then considering the interrelationship. The FFM model posits five major domains of personality: Neuroticism, Extraversion, Conscientiousness, Agreeableness and Openness to Experience, with each factor further defined by six trait facets. Self-report and informant-generated inventories for their assessment were developed by Costa \& McCrae (1992: The Revised NEO Personality Inventory), and a semi-structured interview has recently become available - the Structured Interview for the Five-Factor Model of Personality (SIFFM; Trull \& Widiger, 1997).

Cloninger's Tridimensional Model of Personality proposes the existence of four basic temperaments (Novelty Seeking, Harm Avoidance, Reward Dependence and Persistence) that are held to reflect the putative biological core of personality and to be determined by different neurotransmitter systems. Depending on various psychosocial experiences, character traits (Selfdirectedness, Co-operativeness and Self-transcendence) may be formed, with the PDs then modelled as a combination of extreme temperament scores and low character scores (Svrakic et al. 1993).

The principal alternative approach involves generation of descriptors from patients with a PD. Personality trait structure is viewed as being hierarchical in nature, with specific covarying behaviours viewed as giving rise to narrow band constructs or lower-order traits, which combine to produce several identifiable higher-order traits. Siever \& Davis (1991) so postulated four major dimensions of psychobiological functioning underlying PD behavioural manifestations: anxiety/ inhibition, affective instability, impulsivity/aggression and cognitive/perceptual organization. Livesley et al. (1992) examined personality disorder traits derived from literature review and clinical judgement, with data generating a 290-item self-report measure - The Dimensional Assessment of Personality Pathology-Basic Questionnaire (DAPP-BQ; Schroeder et al. 1992). Analyses (principal components) grouped the 18 identified lower-order traits into four higher-order factors labelled 'Emotional Dysregulation', 'Dissocial Behaviour', 'Inhibitedness' and 'Compulsivity'. Investigations have been consistent in demonstrating stability of traits across both clinical and non-clinical samples (when assessed via self-report), with such consistency being a basic requirement of any dimensional representation of the PDs (Livesley et al. 1998), while conceptual linkages with the first four FFM dimensions underpinning normal personality are evident. Using a similar methodology, Clark (1990) developed a self-report measure (the SNAP or Schedule for Nonadaptive and Adaptive Personality) that assesses 15 basic traits. Comparison of the Livesley et al. and Clark trait sets revealed similar higher-order factor structures (Clark et al. 1996).

It is now widely argued that PD traits represent the extremes of normal personality variation, with, for instance, Widiger et al. (1994) concluding that 'personality disorders are not qualitatively distinct from normal personality functioning, they are simply maladaptive, extreme variants of common personality traits'. If true, both a parsimonious model and simplified measurement are allowed. Similarities between the four higher-order factors and those dimensions agreed to be central in normal personality have been detailed by Livesley (1998). 'Emotional Dysregulation', with its core components of anxiousness and affective lability is described as a general factor that resembles the FFM dimension of Neuroticism. 'Dissocial Behavior', with its core features being callousness and rejection, is said to resemble the negative pole of Agreeableness. 'Inhibitedness', with core features of intimacy problems and a restricted expression of inner experience, is viewed as similar to the pattern of Introversion. Livesley recommends that Compulsivity (which equates with the FFM dimension of Conscientiousness) be coded at the dispositional level, citing the absence of the pervasive effects seen with the other higher-order factors and lack of associated dysfunction as two reasons for this suggestion. The final FFM dimension, 'Openness to experience' does not 'cross walk' readily into any higher-order PD factor.

Some doubts remain about such apparently similar underpinnings to the models. For some, the 
FFM dimensions fail to adequately convey the severe nature of dysfunctional behaviour seen in the PDs. Can for example, the self-mutilation seen in Borderline PD be understood as a maladaptive variant of normal personality traits? The FFM model conceptualizes Borderline Personality Disorder as an extreme variant of Neuroticism, a domain that 'contrasts adjustment or emotional stability with maladjustment' (Costa \& McCrae, 1990). Again, concern is expressed about insufficient sampling of items tapping dysfunctional or extreme levels of personality traits. Widiger \& Costa (1994) acknowledge this possibility in seeking to explain the finding of only moderate or inconsistent support for the hypothesized relationship between Obsessive-Compulsive Personality Disorder (OCPD) and the FFM domain of Conscientiousness.

Others have expressed concern about the underpinning reliance on trait theory, which has dominated research into personality over the years, with the quest being to identify a set of basic traits allowing for a comprehensive description of personality (John, 1990) and of personality disorder. Can the commonly used statistical strategy of factor analysis meet this goal, and is it the method that will lead to the discovery of the underlying structure of personality? Secondly, are delineated 'traits' the appropriate building blocks? Typically, traits are defined as consisting fundamentally of stable internal dispositions, functional units and causal factors within the individual which account for his behaviour across a wide variety of situations. Critics of trait theory highlight several limitations of the method as a tool of enquiry, arguing that it prioritizes dimensions of dispositional behavioural repertoires somewhat at the expense of dispositional thoughts and feelings, and that the focus largely ignores person-environment system interactions. Tellegen (1993) further suggests that what is possibly being studied is 'folk psychology' or beliefs people have about the world rather than the actual structure of personality, while Briggs (1989) has queried whether traits are 'real' or convenient fictions by which we communicate. Thus, the rate-limiting step for some is the development of a sound theoretical model that can provide an underlying explanatory structure. Of course, measurement and modelling do not need to be postponed until the optimal theory is derived. A reciprocal or iterative process, with theory shaping measurement and the converse, will advance the science.

It is also of interest to note a recent study by Pukrup et al. (1998) that aimed to avoid such methodological problems by applying non-metric scaling methods and a facet theoretical analysis in their investigation of the structural relationships between personality factors and disorders. Findings were consistent with recent dimensional models in confirming a continuous transition from normal personality to PD status.

A central question remains, however. If PD traits are merely extremes of normal personality variation, how then do we define personality disorder? It would be easy to adopt a statistical definition based on percentage extremes, but this would be unlikely to satisfy clinicians. We suggest that it might be unwise to preclude the possible identification of a characteristic that more clearly differentiates disordered from normal personality variation. A candidate domain could be to identify and model 'disordered functioning' - and independently (at least in design) from 'personality style' components.

\section{DISORDERED FUNCTIONING: DEFINITION AND MEASUREMENT}

Many current personality descriptors currently confound the two components of personality style (PS) and disordered functioning (DF), raising potentially serious issues for both assessment and measurement. We suggest that the high rates of co-occurrence and overlap consistently found amongst the personality disorders (Pfohl et al. 1986; Joffe \& Regan, 1988) could be a consequence of this confounding. Thus, if PD status is always associated with a certain DF characteristic (say failure to learn from experience), then associations will always be found between individual PDs and between the three PD clusters, because the individual PSs 'share' the inflexible behavioural component - thus driving associations even if the PSs are themselves truly distinct.

Henry (1997) also sees advantage in separation of these components 'for measurement purposes and as well as for the purposes of basic science', while Cloninger (1997) does not agree. He views 
the core nature of disordered functioning as a reason not to separate these components, stating 'functional impairment is a predictable property of both temperament and character, not an independent component of personality'.

Irrespective of such conceptual differences, how could disordered personality functioning be operationalized and measured? As noted, there is debate about how and at what level one defines 'disorder', with levels including the specific surface or behavioural manifestations, as well as underlying traits hypothesized as driving the surface manifestations (e.g. impulsiveness) and cognitive processes. Disordered functioning may also relate less to extreme expression of normal personality traits, and more to the context-inappropriate expression of behaviours.

Livesley et al. (1994) emphasized inflexibility and inadequate performance in 'the universal life tasks of identity, attachment, intimacy and affiliation'. Millon (1986) postulated a number of parameters (i.e. inflexibility, causing personal discomfort, reduction of opportunities, ineffective functioning, non-adjustment to the environment, self-defeating patterns, instability under stress, and causing discomfort to others). Earlier, we noted the DSM-IV requirement for features to lead to clinically significant distress or impairment across several domains, while the old clinical saw (i.e. that such individuals - or those around them-must suffer) identifies an impairment domain. 'Coping' may be a candidate domain - when several studies (e.g. Kruedelbach et al. 1993; Vollrath et al. 1994) have reported that problem-solving strategies (i.e. strategies aimed at the problem causing the distress) are reduced in individuals with a PD.

Livesley (1998) offers a preliminary definition of PD as a tripartite failure involving separate but interrelated realms of functioning: the self-system (the failure to establish stable and integrated representations of oneself and others), familial or kinship relationships/interpersonal relationships (the failure to function adaptively in interpersonal relationships), and societal or group relationships (the failure to develop the capacity for prosocial behaviour and cooperative relationships), while Millon (1986) has argued that PD is expressed across a set of domains (i.e. intimate, peer, family and work relationships, as well as at work). Such an orientation is consistent with the view of Rutter (1987) that a characteristic underpinning many of the categorized PDs is 'a pervasive persistent abnormality in maintaining social relationships'. It also suggests candidate domains for studying disordered functioning.

Once disordered functioning has been modelled and measures developed, relationships between the varying personality styles underpinning the PDs and DF components may assist our overall capacity to model the PDs.

\section{ASSESSMENT}

Assessment of personality and PDs remains difficult despite structured interview formats, particularly reflecting response biases and the influences of state variables (e.g. depression) on trait characteristics (Widiger, 1993) and the role of situational factors in determining the extent to which behaviours can be considered dysfunctional or maladaptive.

The validity of both self-reported and witness-reported data is commonly questioned. A recent survey (Westen, 1997) found, however, that experienced clinical psychologists and psychiatrists regarded observation of the patient's behaviour during interview and descriptions of the patient's interactions with significant others as central to the diagnosis of patients with PDs. This emphasis on 'signs' (phenomena observed by the clinician) over 'symptoms' avoids limitations related to direct questioning about traits, and addresses oft-raised doubts about the validity of self-report data (limited due to defensiveness, lack of insight, etc.). Zimmerman (1994) reviewed studies using informants in the diagnosis of PD, which consistently demonstrated poor agreement between patient and informant, and identified more personality pathology to be reported by the informant.

While these concerns appear reasonable, the usefulness of self-report data remains an empirical question. Of interest here is the work of Funder (1993), who studied 'normal personality', and concluded that individuals can report on their own personality characteristics with reasonable accuracy, as shown by substantial agreement between self-reports and reports by peers and other 
observers. Funder (1987) challenges the consistently reported finding that agreement between selfjudgements of personality and judgements by others is low, claiming that this is so only when the peer raters do not know their subjects well or when unreliable rating scales are used. Moreover, at least four different studies have established that agreement is even higher when the person in question provides a consistent self-description on the trait being evaluated. For Funder (1980) 'if proper care is taken to use valid rating scales and peer judges who know us well, then by and large we do tend to see ourselves as others see us'. We should be less sanguine about such a conclusion, however, for assessing personality disorder traits.

Factors identified that affect inter-rater agreement include: reliability of observer ratings, visibility of the behaviour or trait, how well the informant knows the individual and the social desirability of the trait. In relation to state variable influences, it has been frequently demonstrated that a self-report inventory will provide inflated or deflated (i.e. inaccurate) assessments of personality traits when the subject is depressed, anxious, or psychotic (Widiger, 1993). Thus, PD assessment must examine for and quantify Axis I pathology.

As noted, DSM-IV offers a guide to clinicians looking to distinguish normal personality traits from PDs, suggesting that 'only when personality traits are inflexible and maladaptive and cause significant functional impairment or subjective distress do they constitute personality disorders'. For the individual PDs in DSM-IV, stated criteria do not attempt to identify that point at which personality traits become a PD. While maladaptivity is defined in DSM-IV as 'clinically significant impairment', maladaptivity appears to be a relative concept, potentially present or absent and varying in degree. It is also unlikely that all traits are problematical within all possible circumstances (Leaf et al. 1990). The same degree of tough-minded antagonism that is maladaptive in some situations will be adaptive in others. Those advocating a conceptualization of PD from the perspective of a normal model of personality, emphasize the fact that any one individual will possess a combination of traits, and that any particular trait can have adaptive and maladaptive consequences. Thus, any assessment of maladaptivity needs to consider context variables, whether personal, social or occupational (Widiger, 1993).

Westen \& Shedler (1999), noting such limitations to current assessment measures and techniques, have developed a clinically and empirically derived assessment method, the Shedler-Westen Assessment Procedure or SWAP-200. The SWAP-200 uses the 'Q-Sort' method, an empirically proven method well used in normal personality research. The method relies here on clinicianobserver judgement. The Swap-200 contains 200 personality descriptive statements derived from clinicians' concepts of personality disorder, and which are sorted into eight categories by the clinician-observer as being (at one end) irrelevant or inapplicable to the patient, through to (at the other end) being highly descriptive of the patient. Ratings form a fixed distribution. While items include the DSM-III-R and DSM-IV Axis II criteria, others were drawn from a broad range of alternative clinically-based sources, including existing literature on normal and disordered personality, and clinician feedback. Personality disorder scores measure the match between the description of an individual patient and the diagnostic prototype for a PD. Early reported findings by the authors support the validity of the SWAP-200 as a measure of PD.

\section{AN EVOLUTIONARY PERSPECTIVE}

Livesley (1998) lists six requirements of any empirically-based classification of personality disorder, with one being that classification 'should be theory based'. While we have noted the emerging consensus regarding trait dimensions of importance at both the higher- and lower-order levels, both in normal and disordered personality, no comprehensive theory of personality exists. It is intriguing to pose a Darwinian question - what were the problems that gave rise to disordered personality function as a solution, and what advantages did (or does) such functioning provide?

Evolutionary psychology advances natural selection as the unifying causal process in personality development. For Buss (1995), the relevance of this theory to all psychological mechanisms is both logical and inescapable, because 'all behaviour depends on complex psychological mechanisms, and 
all psychological mechanisms, at some basic level of description, are the result of evolution by selection, then all psychological theories are implicitly evolutionary theories'. For Tooby \& Cosmides (1990) 'animals subsist on information. The single most limiting resource to reproduction is not food or safety or access to mates, but what makes each of them possible: the information required for making adaptive behavioural choices'.

Why have the five broad personality dimensions noted earlier been consistently identified? Any number of pop psychology, psychobiological and alternative reasons could be advanced to argue the evolutionary utility of each dimension, and similarly for their expression at the disordered personality level. For Buss (1995), however, his 'target article' of evolutionary psychology considers why they are so frequently identified empirically, suggesting three ways to approach this question. First, individual differences dictate the amount of success in pursuing certain strategies. Secondly, the five dimensions of personality may summarize the most important features of the social landscape that humans have to adapt to, for 'to know others is an adaptive necessity' (Symons, 1979). Thirdly, such dimensions may signify mere 'noise' in the system - variations that were, in fact, neutral with respect to natural and sexual selection. He goes on to note how the persistent identification of surgency (dominance versus submission) and agreeableness (cooperative versus aggressive) as the two major axes in interpersonal taxonomies 'represents the adaptive significance to all humans of discerning in others their hierarchical position and proclivity and their willingness to form reciprocal alliances'.

This view, of 'personality as an adaptive landscape', argues that perceiving, attending to and acting upon differences in others remains crucial for solving adaptive problems. If valid, a general theory of personality 'must correlate evolved psychological mechanisms, their accompanying behavioural strategies, and the specific adaptive problems they were designed to solve' (Buss, 1991).

For Buss, maladaptive behaviour can be explained in several different ways. For instance, as humans are not 'fitness strivers', such behaviours are due to inappropriate or excessive expression of a propensity that is normally adaptive (posing the further question of why their expression should be excessive). Alternatively, the behaviour was adaptive in an evolutionary context, but is no longer. Another view, and for Hinde (1995) a more useful explanation 'is that the behaviour in question is controlled by the structure of values, norms and beliefs in the culture, which are themselves only distantly related to the propensities of the individual on which selection has acted'. Hinde is more cautious about an evolutionary perspective, calling attention to the difficulties inherent within any definition of adaptiveness, arguing that it is the psychological mechanisms that have been selected not the behaviour(s). Thus, behavioural outcomes can be, but are not necessarily, adaptive as already noted.

Plutchik (1980) identifies the function of personality as the solving of, and adaptation to, major life tasks - with central ones being identity, hierarchy (associated with issues of dominance and submissiveness), territoriality (belongingness) and temporality (problems of loss and separation). Failure to achieve adaptive solutions to one or more of these tasks is viewed as resulting in some of the core PD dysfunction. Convergence of ideas from evolutionary psychology and personality theory creates the possibility that PD can be so defined. Thus, "personality disorder is present when the structure of personality prevents the person from achieving adaptive solutions to the universal life tasks of establishing a self-system, attachment and intimacy, and cooperativeness and prosocial behaviour' (Livesley, 1998). Such a definition assumes that PD results from the failure to acquire the structures to function effectively in these realms. Another possibility is that the individual has acquired the necessary structures but is directing his behaviour in less than adaptive ways.

\section{GENETIC STUDIES}

Research suggests that genetic factors account for nearly half the variance in explaining individual differences in personality traits (Plomin et al. 1997). That environmental factors exert a greater than equal influence indicates 'that although temperamental factors bend the twig, they do not, by themselves determined the shape of the entire tree' (Silk, 1998). 
Accepting the view that there is continuity between normal and abnormal traits, and using a 'trait and type' methodology, the PDs are increasingly being viewed as 'pathological amplifications of normal traits' (Paris, 1996), with various combinations of traits representing the diatheses that determines what type of PD any individual will develop. Research may then be expected to determine those specific combinations of traits most likely to lead to personality disordered functioning.

For a lengthy period, genetic studies tended to concentrate on the broad dimensions of extroversion and neuroticism. Their high heritability has prompted the suggestion that the heritability of lower order traits merely reflects that they are components of these more general traits. Earlier, we noted the study by Livesley et al. (1998), which analysed DAPP-BQ scores and, across three differing samples, identified a four-factor 'phenotypic' structure (i.e. 'Emotional Dysregulation', 'Dyssocial', 'Inhibition' and 'Compulsivity') across each sample. Multivariate genetic analyses also identified four 'genetic' and 'environmental' factors that 'were remarkably similar to the phenotypic factors'. In the sample of volunteer twins, hereditability estimates were derived for scores on the DAPP-BQ scales (following removal of the effects of the four higher-order components), with correlations higher on all 'traits' for MZ than for DZ twins. The authors interpreted that finding as overthrowing the common interpretation that any hereditability of lowerorder traits merely reflects their being components of more general traits such as neuroticism and extraversion. They argued that genetic influences appear to act via 'multiple specific predispositions and a few general factors'.

Similar findings, showing substantial residual variance when the effects of the higher-order dimensions are removed, have been reported for the specific facet traits of the FFM. For the dimension of neuroticism, for example, the residual component heritability for the six facets ranged from $21 \%$ to $29 \%$ (Jang et al. 1998). These findings have far-reaching implications for our understanding of personality and PD. If, as detailed earlier, the PDs are extreme expressions of normal personality traits, we can conceptualize a large number of genetic building blocks, each having a relatively specific effect in isolation, but also with the potential to combine to produce an almost infinite variety of configurations of personality traits and able to be expressed as "personality disorder'.

\section{CONCLUSIONS}

Increasingly, 'personality' is viewed as higher and lower dimensions existing on a continuum, with no sharp division between normality and pathology. Thus, the PDs can be understood as representing extreme variants of normal traits. Empirical studies provide strong support for a dimensional PD model, with multiple distinct genetic factors influencing end-point expression. The distinct categories suggested within classification guides like DSM-IV are therefore unlikely to exist. Nevertheless, such classification systems have been major influences in framing current research agendas and questions, in that the lower-order traits identified and analysed in recent studies identifying the 'continuum' have often been derived from DSM diagnostic criteria.

Although a framework for classifying personality and personality disorders is emerging, the fact is that no comprehensive theory for understanding the personality disorders has yet been formulated. While an evolutionary perspective offers promise, its value will depend on the ability to articulate more precisely the correlations between selected evolved mechanisms, like the lowerorder traits, and their adaptive purpose.

Where does all this leave the clinician? Adoption of a dimensional model may allow assessment and management to proceed with less clinical error, and respect the consensus that has emerged in favour of a dimensional classificatory system.

Equally for researchers, assessment would then be concerned less with formal 'diagnoses' and more with the measurement of individual differences on relevant trait dimensions. Different trait profiles could then be investigated from a stress diathesis perspective, while treatment might address the reduction of vulnerability and the fostering of greater protective and coping potential. A 
downside is the loss to the clinician of categorical assignment, which, at best, can offer ease and a meaningful lexicon in formulating a 'case', in briefing colleagues and in considering management plans.

Future research offers the promise of further definitions of personality and personality disorder, thus assisting understanding of biological determinants and, of particular interest, their genetic underpinnings, and whether character traits and disordered functioning components are as heritable as personality traits. Taken together, such gridlines could better 'place' an individual and assist the clinician to comprehend the emotional and social landscape of that individual, whether disordered or functional.

GORDON PARKER AND ELAINE BARRETT

We acknowledge funding from NHMRC Program Grant (993208) and a NSW Health Department Infrastructure Grant Program to the Mood Disorders Unit. We wish to thank Robert Finlay-Jones and Dusan Hadzi-Pavlovic for discussions prompting this paper, and Kerrie Eyers for manuscript assistance.

\section{REFERENCES}

American Psychiatric Association (1994). Diagnostic and Statistical Manual of Mental Disorders. American Psychiatric Association: Washington, DC.

Briggs, S. R. (1989). The optimal level of measurement for personality constructs. In Personality Psychology: Recent trends and Emerging Direction (ed. D. M. Buss and N. Cantor), pp. 246-260. SpringerVerlag: New York.

Buss, D. M. (1991). Evolutionary personality psychology. Annual Review of Psychology 42, 459-492.

Buss, D. M. (1995). Evolutionary psychology: a new paradigm for psychological science. Psychological Inquiry 6, 1-30.

Clark, L. A. (1990). Toward a consensual set of symptom clusters for assessment of personality disorder. In Advances in Personality Assessment, Vol. 8 (ed. J. Butcher and C. Spielberger), pp. 243-266. Erlbaum: Hillsdale, NJ.

Clark, L. A., Livesley, W. J., Schroeder, M. L. \& Irish, S. L. (1996). Convergence of two systems for assessing personality disorder. Psychological Assessment 8, 294-303.

Cloninger, C. R. (1987). A systematic method for the clinical description and classification of personality variants. Archives of General Psychiatry 44, 573-588.

Cloninger, C. R. (1997). Special feature: etiology of personality disorders: a commentary on Dr Parker's tripartite model. Journal of Personality Disorders 11, 370-374.

Costa, P. T. \& McCrae, R. R. (1990). Personality disorders and the five-factor model of personality. Journal of Personality Disorders $\mathbf{4}$, 362-371.

Costa, P. T. \& McCrae, R. R. (1992). Revised NEO Personality Inventory (NEO PI-R) and NEO Five Factor Inventory (NEO-FFI) Professional Manual. Psychological Assessment Resources: Odessa, FL

Funder, D. C. (1980). On seeing ourselves as others see us: self-other agreement and discrepancy in personality ratings. Journal of Personality 48, 473-493.

Funder, D. C. (1987). Errors and mistakes: evaluating the accuracy of social judgement. Psychological Bulletin 101, 75-90.

Funder, D. C. (1993). Judgements as data for personality and developmental psychology: error versus accuracy. In Studying Lives Through Time: Personality and Development (ed. D. C. Funder, R. Parke, C. Tomlinson-Keasey and K. Widamen), pp. 121-146. American Psychological Association: Washington, DC.

Henry, W. P. (1997). Conceptual issues in measuring personality disorder change. In Measuring Patient Changes in Mood, Anxiety and Personality Disorders: Toward a Core Battery (ed. H. H. Strupp, L. M. Horowitz and M. J. Lambert), pp. 461-488. American Psychological Association: Washington, DC.
Hinde, R. A. (1995). The adaptionist approach has limits. Psychological Inquiry 6, 50-53.

Jang, K. L., McCrae, R. R., Angleitner, A., Rieman, R. \& Livesley, W. J. (1998). Heritability of facet-level traits in a cross cultural twin study: support for a hierarchical model of personality. Journal of Personality and Social Psychology 74, 1556-1565.

Joffe, R. J. \& Regan, J. J. (1988). Personality and depression. Journal of Psychiatric Research 4, 279-286.

John, O. P. (1990). The 'Big Five' factor taxonomy: dimensions of personality in the natural language and in questionnaires. In Handbook of Personality (ed. L. A. Pervin), pp. 66-100. Guilford Press: New York.

Kruedelbach, N., McCormick, R. A., Schulz, S. C. \& Grueneich, R. (1993). Impulsivity, coping styles, and triggers for craving in substance abusers with borderline personality disorders. Journal of Personality Disorders 7, 214-222.

Leaf, R. C., DiGiuseppe, R., Ellis, A., Mass, R., Back, W., Wolfe, J. \& Alington, D. E. (1990). 'Healthy' correlates of MCMI scales 4, 5, 6 and 7. Journal of Personality Disorders 4, 312-328.

Leary, T. (1957). Interpersonal Diagnosis of Personality. Raven: New York.

Livesley, W. J. (1998). Suggestions for a framework for an empirically based classification of personality disorder. Canadian Journal of Psychiatry 43, 137-147.

Livesley, W. J., Jackson, D. N. \& Schroeder, M. L. (1992). Factorial structure of traits delineating personality disorders in clinical and general population samples. Journal of Abnormal Psychology 101, 432-440.

Livesley, W. J., Schroeder, M. L., Jackson, D. \& Jang, K. L. (1994). Categorical distinction in the study of personality disorder: implications for classification. Journal of Abnormal Psychology 103, 6-17.

Livesley, W. J., Jang, K. L. \& Vernon, P. A. (1998). Phenotypic and genetic structure of traits delineating personality disorder. Archives of General Psychiatry 55, 941-948.

Millon, T. (1986). Personality prototypes and their diagnostic status. In Contemporary Directions in Psychopathology. Toward the DSMIV (ed. T. Millon and G. I. Klerman) pp. 671-712. Guilford Press: New York.

Nestadt, G., Romanoski, A. J., Samuels, J. F., Folstein, M. F. \& McHugh, P. R. (1992). The relationship between personality and DSM-III Axis I disorders in the population: results from an epidemiological survey. American Journal of Psychiatry 149, $1228-1233$.

Paris, J. (1996). Social Factors in Personality Disorders. Cambridge University Press: New York.

Parker, G., Roussos, J., Wilhelm, K., Mitchell, P., Austin, M.-P. \& Hadzi-Pavlovic, D. (1998). On modelling personality disorders: 
are personality style and disordered functioning independent or interdependent constructs? Journal of Nervous and Mental Disease 186, 709-715.

Pfohl, B., Coryell, W., Zimmerman, M. \& Stangl, D. (1986). DSMIII personality disorders: diagnostic overlap and internal consistency of individual DSM-III criteria. Comprehensive Psychiatry 27, 21-34.

Plomin, R., DeFries, J. C., McClearn, G. E. \& Rutter, M. (1997). Behavioral Genetics. Freeman: New York.

Plutchik, R. (1980). Emotion: A Psychoevolutionary Synthesis. Harper \& Row: New York.

Pukrop, R., Herpertz, S., Henning, S. \& Steinmeyer, E. M. (1998). Special feature: Personality and personality disorders. A facet theoretical analysis of the similarity relationships. Journal of Personality Disorders 12, 226-246.

Rutter, M. (1987). Temperament, personality and personality disorder. British Journal of Psychiatry 150, 443-458.

Schroeder, M. L., Wormworth, J. A. \& Livesley, W. J. (1992). Dimensions of personality disorder and their relationship to the big five dimensions of personality. Psychological Assessment 4, 47-53.

Shea, M. T. (1995). Interrelationships among categories of personality disorder. In The DSM-IV Personality Disorders (ed. W. J. Livesley), pp. 397-406. Guilford Press: New York.

Siever, L. J. \& Davis, K. L. (1991). A psychobiological perspective on personality disorders. American Journal of Psychiatry 148, 1647-1658.

Silk, K. R. (1998). Biology of Personality Disorders. American Psychiatric Press: Washington DC.

Svrakic, D. M., Whitehead, C., Przybeck, T. R. \& Cloninger, C. R. (1993). Differential diagnosis of personality disorders by the seven factor model of temperament and character. Archives of General Psychiatry 50, 991-999.

Symons, D. (1979). The Evolution of Human Sexuality. Oxford University Press: New York.

Tellegen, A. (1993). Folk concepts and psychological concepts of personality and personality disorder. Psychological Inquiry 4, $122-130$.
Tooby, J. \& Cosmides, L. (1990). On the universality of human nature and the uniqueness of the individual: the role of genetics and adaptation. Journal of Personality 58, 17-68.

Trull, T. J. \& Widiger, T. A. (1997). Structured Interview for the FiveFactor Model of Personality (SIFFM): Professional Manual. Psychological Assessment Resources: Odessa, FL.

Vollrath, M., Alnaes, R. \& Torgersen, S. (1994). Coping and MCMIII personality disorders. Journal of Personality Disorders 8, 53-64.

Westen, D. (1997). Divergences between clinical and research methods for assessing personality disorders: implications for research and the evolution of Axis II. American Journal of Psychiatry 154, 895-903.

Westen, D. \& Shedler, J. (1999). Revising and assessing Axis II, Part I: developing a clinically and empirically valid assessment method. American Journal of Psychiatry 156, 258-272.

Widiger, T. A. (1992). Categorical versus dimensional classification: implications from and for research. Journal of Personality Disorders 6, 287-300.

Widiger, T. A. (1993). The DSM-III-R categorical personality disorder diagnoses: a critique and an alternative. Psychological Inquiry 4, 75-90.

Widiger, T. A. \& Costa, P. T. (1994). Personality and personality disorders. Journal of Abnormal Psychology 103, 78-91.

Widiger, T. A., Trull, T. J., Clarkin, J. F., Sanderson, C. J. \& Costa, P. T. (1994). A description of the DSM-III-R and DSM-IV personality disorders with the Five-Factor model of personality. In Personality Disorders and the Five-Factor Model of Personality (ed. P. T. Costa and T. A. Widiger), pp. 41-56. American Psychological Association: Washington, DC.

Wiggins, J. (1982). Circumplex models of interpersonal behaviour in clinical psychology. In Handbook of Research Methods in Clinical Psychology (ed. P. Kendall and J. Butcher), pp. 183-221. Wiley: New York

Zimmerman, M. (1994). Diagnosing personality disorders: a review of issues and research methods. Archives of General Psychiatry 51, 225-245.

Zimmerman, M. \& Coryell, W. H. (1990). DSM-III personality disorder dimensions, Journal of Nervous and Mental Disease 178, 686-692. 